\title{
Novel Multi-Scale Filter Profile-Based Framework for VHR Remote Sensing Image Classification
}

\author{
Zhiyong Lv ${ }^{1}$, Guangfei $\mathrm{Li}^{1}$, Yixiang Chen ${ }^{2, *}$ and Jón Atli Benediktsson ${ }^{3}(\mathbb{C}$ \\ 1 School of Computer Science and Engineering, Xi'An University of Technology, Xi' an 710048, China; \\ zhiyonglyu@xaut.edu.cn (Z.L.); liguangfei_dream@hotmail.com (G.L.) \\ 2 Department of Surveying and Geoinformatics, Nanjing University of Posts and Telecommunications, \\ Nanjing 210023, China \\ 3 Faculty of Electrical and Computer Engineering, University of Iceland, Reykjavik IS 107, Iceland; \\ benedikt@hi.is.s \\ * Correspondence: chenyixiang@njupt.edu.cn; Tel.: +86-158-2967-3435
}

Received: 31 July 2019; Accepted: 11 September 2019; Published: 16 September 2019

\begin{abstract}
Filter is a well-known tool for noise reduction of very high spatial resolution (VHR) remote sensing images. However, a single-scale filter usually demonstrates limitations in covering various targets with different sizes and shapes in a given image scene. A novel method called multi-scale filter profile (MFP)-based framework (MFPF) is introduced in this study to improve the classification performance of a remote sensing image of VHR and address the aforementioned problem. First, an adaptive filter is extended with a series of parameters for MFP construction. Then, a layer-stacking technique is used to concatenate the MPFs and all the features into a stacked vector. Afterward, principal component analysis, a classical descending dimension algorithm, is performed on the fused profiles to reduce the redundancy of the stacked vector. Finally, the spatial adaptive region of each filter in the MFPs is used for post-processing of the obtained initial classification map through a supervised classifier. This process aims to revise the initial classification map and generate a final classification map. Experimental results performed on the three real VHR remote sensing images demonstrate the effectiveness of the proposed MFPF in comparison with the state-of-the-art methods. Hard-tuning parameters are unnecessary in the application of the proposed approach. Thus, such a method can be conveniently applied in real applications.
\end{abstract}

Keywords: remote sensing imagery; multi-scale filter profiles; very high resolution; land cover classification

\section{Introduction}

A remote sensing image with very high resolution (VHR) has an improved visual appearance over imagery of conventional resolution [1]. The remote sensing images with VHR mean that the size of per pixel in the image is smaller than that of the low-median resolution image. Therefore, the remote sensing image with VHR can capture and describe the details of ground targets in terms of size and shape. Such imagery plays an important role in various practical applications, such as land cover classification [2-4], target recognition [5-7], and change detection [8,9]. However, VHR remote sensing images usually have insufficient spectral information, that is, their numbers of spectral bands are lacking. The spectral bands for most high-resolution remote sensing sensors are less than eight. For example, Quick Bird satellite image contains four spectral bands, and WorldView-3 image only has eight multi-spectral bands $[10,11]$. This situation is attributed to remote sensors, which have physical limitations between spatial and spectral resolutions. Thus, the high resolution in geometry and low deliverance in spectral reflectance produce the Hughes phenomenon and cause considerable noise in the classification map [12-16]. 
A filter is used to address this problem. Filtering is an intuitive yet simple way of smoothing the noise of a VHR image. The classical methods, such as mean filtering (MF) [17] and median filtering (MedF) [18], have been applied to reduce the noise in images and signals $[19,20]$. Several spatial filters have been developed for VHR remote sensing image classification [21,22]. For example, the modified mean filter (MMF), which can smooth the central pixel value using the mean of its adaptive region, was proposed to improve the classification performance of VHR remote sensing images [23]. In addition to image filters, spatial-spectral feature complement is another method for enhancing the classification performance while using VHR remote sensing images. For example, an attribute profile (AP) feature based on morphology is extended with multi-scale parameters to consider the uncertainty of a ground target in size and shape [24]. Moreover, the AP morphology is extended with multi-shape structural elements (M_EMPs) for image classification [25]. Additionally, edge-preserving filters (EPFs) [26] and recursive filters (RFs) [27] have been proposed to extract spatial features from high-resolution image classification, and a rolling guidance filter (RGF) has been developed to smooth the internal noise of a target and maintain the edge among different targets [28]. In brief, using spatial-spectral features has been verified to be effective in improving the performance of VHR remote sensing images [29]. In addition, CNN has been widely used for land cover classification with remote sensing image [30-32]. Although the developed algorithms can improve the classification performance and accuracies with VHR remote sensing images, these algorithms are complex. Such complexity can be considered a problem because the parameter setting of these methods is required for real application. Clearly, no available method has been labeled as "the appropriate one" for all classification cases $[33,34]$. Therefore, the classification performance in the aforementioned case must be improved while maintaining the usability of the method for practical engineering applications.

In recent years, post-processing has been verified as effective in further improving the classification of VHR remote sensing images. Tang et al. presented a multiple-point geostatistical simulation for post-processing land cover classification with a remote sensing image [35]. Huang et al. conducted a systematic study of new post-processing methods for image classification [36]. In the previous study, two post-processing methods were proposed to improve the classification performance on VHR images $[37,38]$. In these studies, the post-processing technique is effective in smoothing the noise of the initial classification map and improving the performance and accuracy results. However, the preand post-processing for acquiring the initial classification map is independent. This operation requires the familiarization of practitioners with the pre- and post-processing approaches.

In the present study, multi-scale filter profiles (MFPs) are proposed to extract the multi-scale spatial-spectral feature without hard-tuning parameters. This task is initiated to improve the intra-class homogeneity in VHR remote sensing images. Moreover, the spatial adaptive region of MPFs is extensively applied to smooth the noise of the initial classification map further. The proposed MPF-based framework (MFPF) is the first approach to fuse image pre-processing and post-classification processing. This integration enhances the usability and convenience of the proposed approach. The major contributions of the proposed method are presented as follows:

(1) MMF [23] is first extended with multi-scale parameters to construct MFPs. Experimental comparison has demonstrated that this extension is helpful and effective for improving classification performance [20]. In the present study, the proposed MFPs not only improve the homogeneity of a target but also the separability of the ground target under consideration. This feature is attributed to the substantial scale information of the target that is adopted and utilized through the expanding values of the parameter $T_{1}$.

(2) The parameter setting of the proposed MFPs is easy for practical applications and requires no parameter hard-tuning for different datasets. In the experiments with different datasets, the proposed MFPF is effective in terms of classification accuracies for different datasets without parameter tuning.

(3) The proposed approach is the first to integrate the pre- and post-classification techniques through multi-scale filters. This integration of the entire technology is beneficial for promoting the application of the proposed MPFs. The serial value of $T_{1}$ not only extends the coverage of spectra but 
also utilizes the multi-scale information in the spatial domain. The adaptive regions for the multi-scale filter are used for further post-processing of the initial classification map.

The remainder of this paper is organized as follows. Section II discusses the details of the proposed approach. Section III provides the experiments and comparisons. Finally, Section IV presents the conclusion drawn from this study.

\section{Proposed Method}

This section introduces the details of the proposed MFPF for classification with VHR remote sensing images. Figure 1 illustrates that the proposed MFPF comprises the following parts. First, MFPs are developed, and a layer-stacking technique is used to fuse the multi-scale filtered image profiles. Then, principal component analysis (PCA) is adopted to reduce redundancy. Finally, an initial classification map is acquired through a supervised classifier, and a post-classification method is proposed on the basis of the MFs. Each part is detailed as follows.

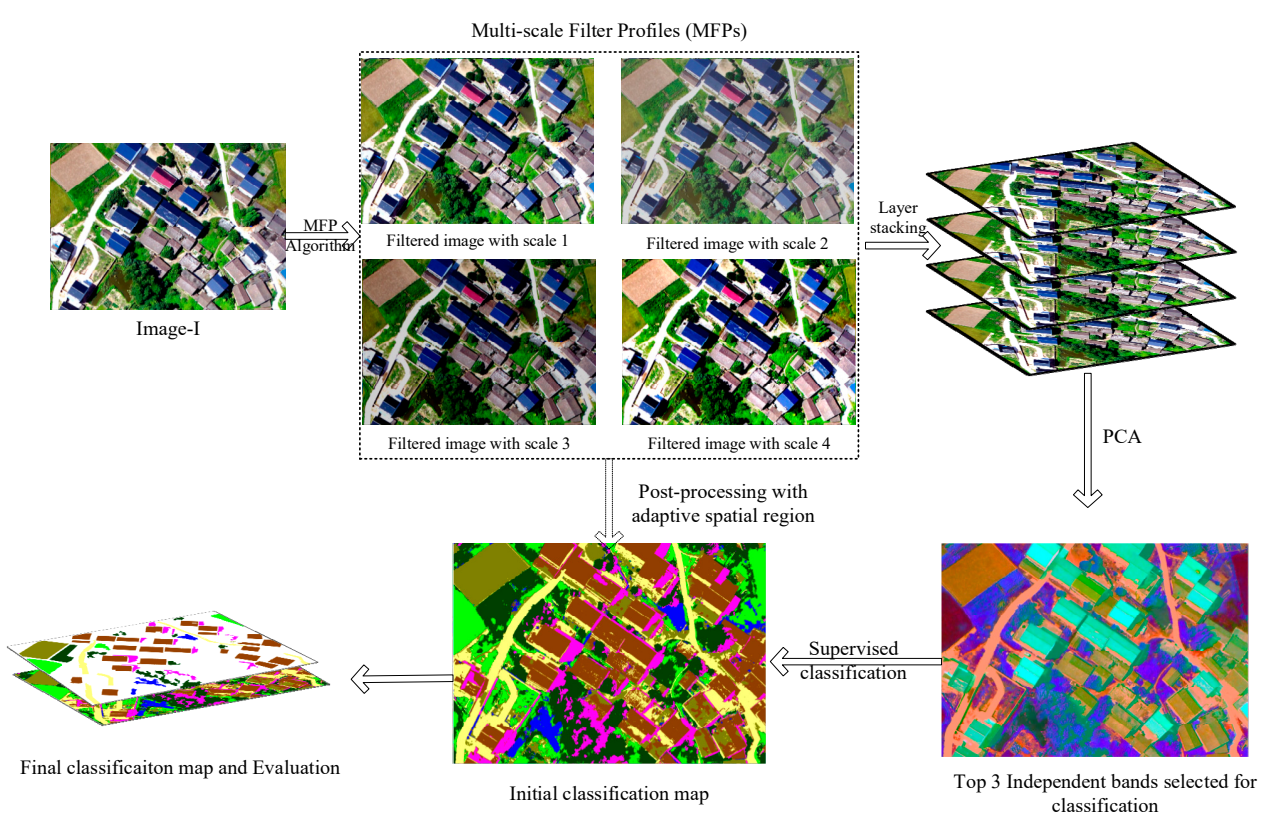

Figure 1. Flowchart of the proposed MFPF.

\subsection{Generation of MFPs}

In the previous method (MMF) [23], parameter " $T_{1}$ " is the measured spectral difference between the central pixel and its neighbors, and $T_{2}$ is the size of an adaptive extension and is insensitive to the classification accuracies. By contrast, $T_{1}$ is sensitive to the classification accuracy, and selecting $T_{1}$ for a given image is time consuming and depends on the practitioner's experience. The idea of parameter serialization is introduced in this study to handle the limitation of the previous MMF and avoid the hard-tuning of $T_{1}$ for the classification of different datasets.

In the proposed MFPF, $T_{1}$ is serialized with five values with a fixed $T_{2}$, such as $T_{1}=\left\{S_{1}, S_{2}, S_{3}, S_{4}, S_{5}\right\}$, where $S_{k}$ indicates the different values of $T_{1}$. The adaptive extension algorithm in the previous study [23] indicates that a different $T_{1}$ with a fixed $T_{2}$ will create the extended regions with various shapes. This phenomenon is attributed to the different $T_{1}$ values, which will change the searching direction in the extension process. In addition, different searching directions will affect the shape of the adaptive extension region. Therefore, the multi-scale feature of a target will be described and utilized when $T_{1}$ is set to different values. 
Herein, "I" is assigned as a VHR remote sensing image with an R-G-B band false color. An adaptive extended region with a pair of $T_{1}$ and $T_{2}$ is used for smoothing each band of the given image (I) with the mean of the adaptive region to construct MFPs. Thus, the formula of MFPs can be written as

$$
\mathrm{MPFs}=\left\{\mathrm{I}_{\mathrm{S}_{1}}, \mathrm{I}_{\mathrm{S}_{2}}, \mathrm{I}_{S_{3}}, \mathrm{I}_{S_{4}}, \mathrm{I}_{S_{5}}\right\},
$$

In the equation-1, where $I_{S_{1}}$ is the filtered image with the pair of $T_{1}=s_{1}$, and $T_{2}$ is fixed at a pre-provided value. $I_{s_{1}}=\left\{I_{r}^{S_{1}}, I_{g}^{S_{1}}, I_{b}^{S_{1}}\right\}$, where " $r$, g, and b" denote the red, green, and blue bands,

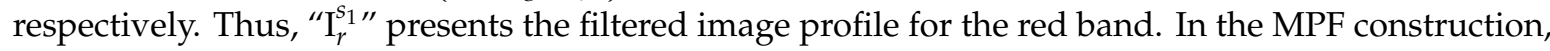
MPFs are the filtered profiles, which are developed by smoothing the raw image band-by-band with the adaptive extension algorithm using different values of parameter $T_{1}$ and the fixed value of $T_{2}$.

\subsection{Reduction of Redundancy through PCA}

MPFs can capture the multi-scale feature of a ground target but is redundant in the data construction of a target. In this section, a classical method called PCA is proposed to reduce redundancy. Before applying the PCA, a layer-stacking method, which is embedded in the ENVI 5.2 professional software, is adopted to fuse the MPFs into a high-dimensional vector.

The details of PCA are not introduced in this study because it is a classical method that has been successfully applied to many image-processing cases [39-41]. In the proposed MFPF, the PCA application tool, which is integrated into a toolbox of the ENVI 5.2 business software, is utilized to reduce the dimension of the high-dimensional vector. An observation is depicted in Figure 2 to demonstrate the capability of the proposed approach further in improving the intra-class homogeneity and preserving the edge of different classes. The observations and comparison results demonstrate that the smallest standard deviation of each band is achieved through the proposed approach. The boundary of the ground targets is preserved, as demonstrated by the yellow arrow in the figures.

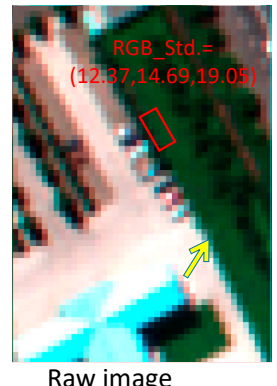

(a)

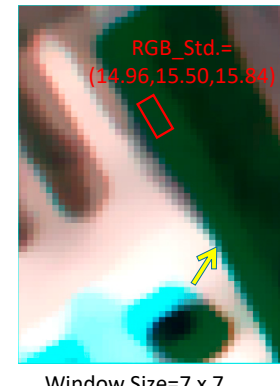

(b)

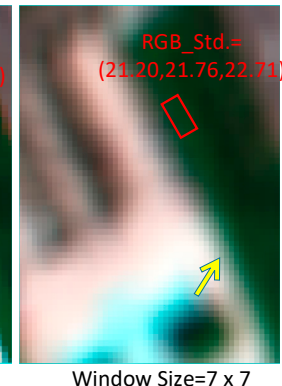

(c)

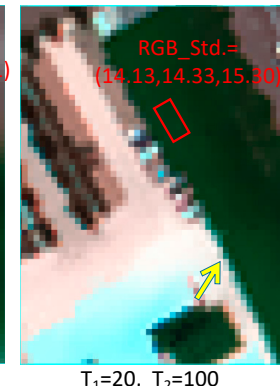

(d)

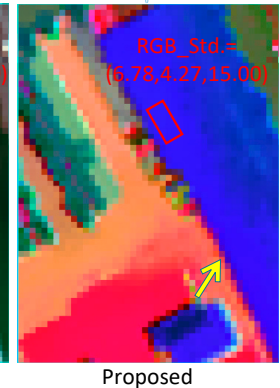

(e)

Figure 2. Comparison between the filtered images and the different methods ("RGB_Std." represents the standard deviation value for R-G-B). (a) raw image with a $1.0 \mathrm{~m} /$ pixel resolution; (b), (c), and (d) are the images filtered by MF, MedF, and MMF, respectively; (e) image comprising the top three components of MPFs with PCA.

The reduced dimension feature vector is considered the "input feature" of a supervised classifier, such as support vector machine (SVM). The initial classification map is acquired.

\subsection{Post-Processing Classification with MFs}

As previously mentioned, each filter in the applied MFs has a corresponding adaptive spatial region. In this section, the label of each pixel in the initial classification map is revised in accordance with the labels of its neighbors. A post-processing classification approach is proposed on the basis of the MFPs. First, the label of each class around a pixel at position $(i, j)$ is counted. If $N_{i j}^{C_{m}}$ denotes the total number of the label for the specific class $C_{m}$, then $N_{i j}^{C_{m}}$ is obtained using Equation (2). Second, the label of the central pixel at position $(i, j)$ is revised using the class that frequently appears in the multi-scale 
adaptive regions, as expressed in Equation (3). The label $\left(L_{i j}\right)$ of the pixel at $(i j)$ is determined by the label of the frequently appearing class.

$$
\begin{aligned}
& N_{i j}^{C_{m}}=R_{c_{m}}^{S_{1}}+R_{C_{m}}^{S_{2}}+R_{C_{m}}^{S_{3}}+\ldots,+R_{C_{m}}^{S_{k}} \\
& L_{i j}=\operatorname{argmax}\left\{N_{i j}^{C_{0}}, N_{i j}^{C_{1}}, N_{i j}^{C_{2}}, \ldots, N_{i j}^{C_{M}}\right\}
\end{aligned}
$$

where $R_{\mathcal{c}_{m}}^{S_{1}}$ is the number of pixels for special class $C_{m}$ within the adaptive region at scale $s_{1}$, " $k$ " is the total number of scale parameter that is equal to the number of different values of $T_{1}$, " $M$ " is the total number of classes for a given image scene, and " $m$ " is the number of counted classes.

This post-processing with multi-scale adaptive regions can further smooth intra-class noise and preserve the boundary between different classes. These features are attributed to the pixels, which comprise a target that is generally homogeneous in spectra and continuous in the spatial domain. The shape and size of a target in the remote sensing are unknown before classification. Thus, the use of a multi-scale adaptive shape of the MFs in the post-processing of the initial classification map should be helpful for smoothing noise and improving classification performance as demonstrated by the experiments performed in this study.

\section{Experiments}

In this section, three experiments are conducted with three real VHR remote sensing images to verify the effectiveness and superiority of the proposed MFPF. The experimental details are presented as follows.

\subsection{Datasets}

(1) JX01: This dataset was acquired using a Canon EOS 5D Mark II camera banded with an unmanned aerial vehicle (UAV) platform. The flight height is approximately $100 \mathrm{~m}$, and the spatial resolution is $0.1 \mathrm{~m} /$ pixel. This image covers a countryside scene in Jiang Xi City, China, and the scene size is $1400 \times 1000$ pixels. Figure 3 exhibits a typical area in the countryside of China. Seven classes, including roads, grass, buildings, shadow, trees, soil, and water, were identified in this scene.

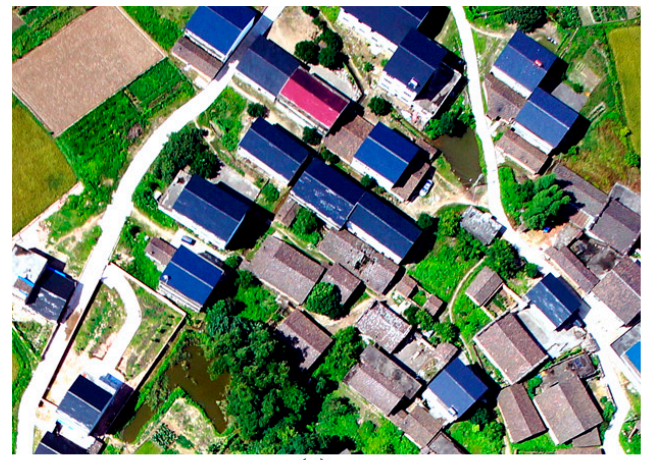

(a)

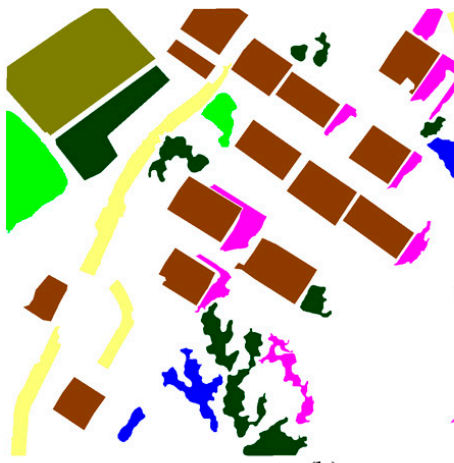

(b)

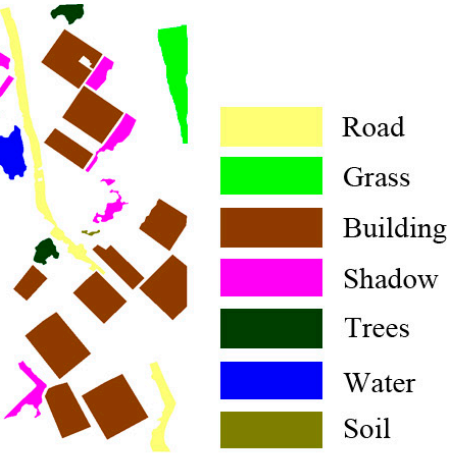

Figure 3. JX01 countryside area, Jiang Xi City, China. (a) Three-band false-color image, (b) ground reference map and class legend.

(2) Pavia University: This dataset is obtained using a ROSIS-03 sensor with a spatial resolution of $1.0 \mathrm{~m} /$ pixel. The original data contain 103 bands with $610 \times 340$ pixels. In this experiment, bands 010 , 27 , and 46 display red, green, and blue, correspondingly (Figure $4 \mathrm{~b}$ ). The ground reference map and legend demonstrate the nine interesting classes. 


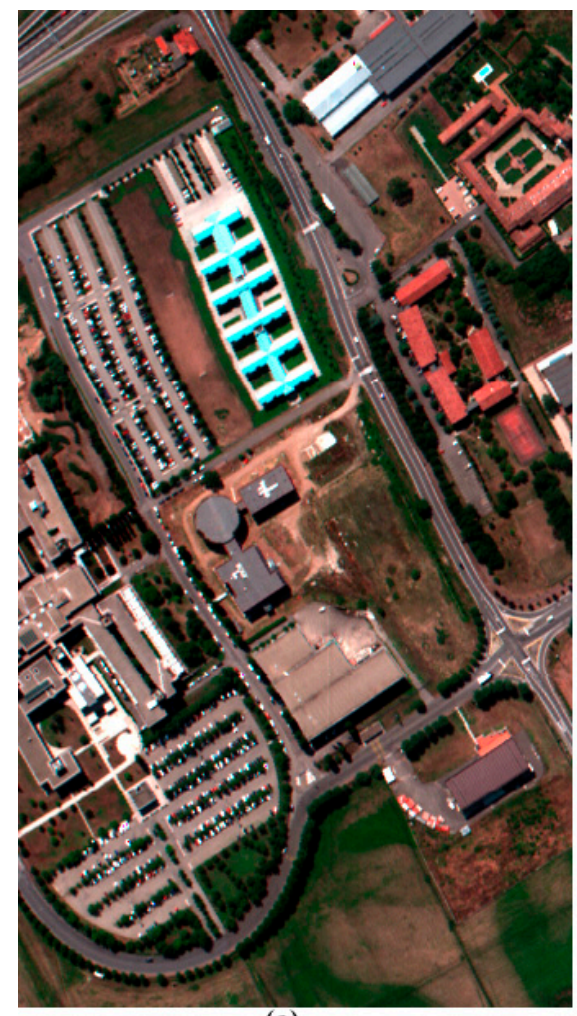

(a)

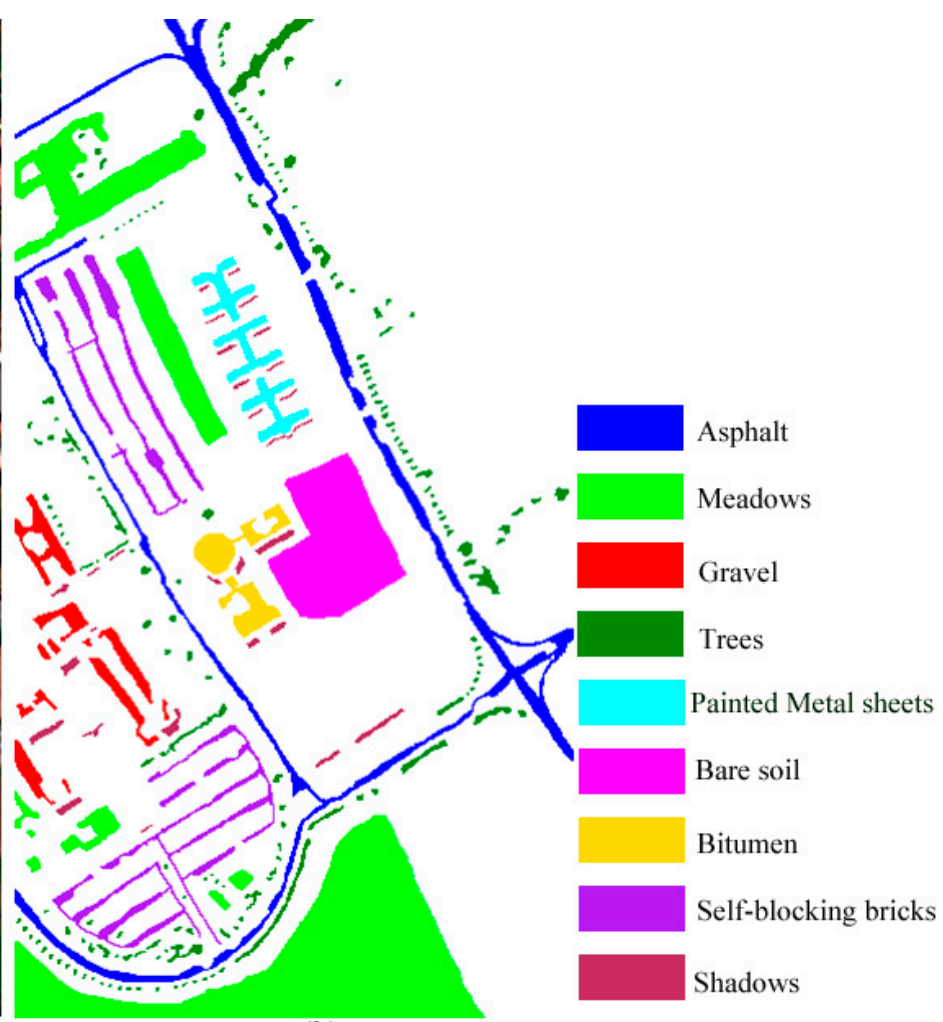

(b)

Figure 4. Pavia University image. (a) Three-band false-color image, (b) ground reference map and class legend.

(3) ZH-6 of Zurich_dataset_v1.0: The third dataset comprises four channels, namely, NIR, R, G, and $\mathrm{B}$, with a resolution of $0.62 \mathrm{~m} /$ pixel. The size of the ZH-6 dataset is $984 \times 812$ pixels, and seven interesting classes are referenced for classification, as shown in Figure 5.

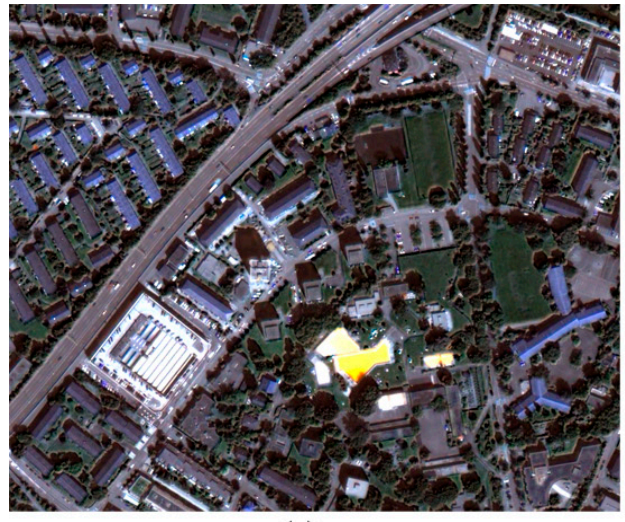

(a)

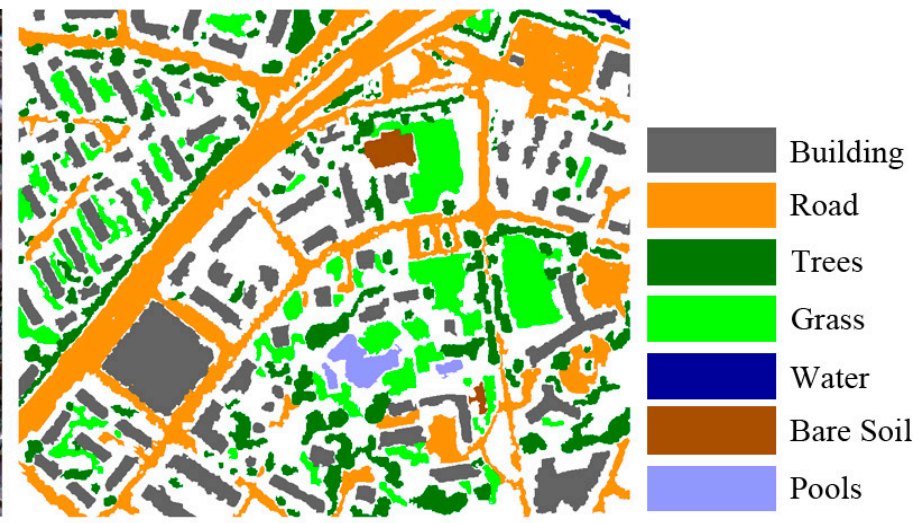

(b)

Figure 5. ZH-6 dataset of the urban area in Zurich, Switzerland. (a) Three-band false-color image, (b) ground reference map and class legend.

\subsection{Experimental Setting}

Three experiments were conducted in this section to test the effectiveness of the proposed MFPF for classifying VHR remote sensing images. 
(1) In the first experiment, the JX01 dataset is adopted and classified using the SVM classifier, which is an extensively used pixel-wise classifier. The proposed MFPF is compared with the classification of raw and filtered images based on MF, MedF, and MMF [23].

(2) In the second experiment, three classical and extensively used supervised classifiers, namely, SVM, k-nearest neighbor (KNN), and Random Tree (RT), are adopted to classify the Pavia University image scene. This task is initiated to test the robustness of the proposed approach further in terms of the different classifiers. The effectiveness of the proposed MFPF is further verified by comparing the different filters in terms of the three classifiers.

(3) In the third experiment, based on the ZH-6 dataset, the adaptability of the proposed MFPF is further investigated by comparing several popular spatial-spectral feature-based approaches. Herein, the following four extensively used spatial-spectral feature-based methods and their corresponding parameter settings are used: EPFs [26]: $\delta_{s}=4, \delta_{r}=0.2, \mathrm{r}=4, \in=0.01$; RFs [27]: $\delta_{s}=200, \delta_{r}=30$, iteration $=3$; M_EMPs [25]: SE $=\{$ "disk", "line", "square", "diamond" $\}$, size $=4 \times 4 ; \operatorname{RGF}[28]: \delta_{s}=4, \delta_{r}=0.1$, iteration $=5$.

In all the experiments, the parameters of the proposed MFPF are fixed at $T_{1}=\{10,15,20,25,30\}$ and $T_{2}=100$ without hard-tuning. Three extensively used indexes, namely, overall accuracy (OA), average accuracy (AA), and the kappa coefficient (Ka), are used to evaluate the performance of the proposed methods. OA is the total number of correctly classified samples divided by the total number of test samples, AA represents the mean value of correctly classified pixels for each class compared with each test sample, and Ka indicates the inter-rater reliability for the classified result [42]. The parameters of each approach are optimized through a trial-and-error method for a specific dataset to guarantee the fairness of comparison.

\subsection{Experimental Results}

In the first experiment based on the JX01 dataset, Table 1 displays that each filter improves the classification accuracies in comparison with those of the raw image without any filter processing. For example, the improvements of OA, AA, and Ka are $4.12 \%, 2.99 \%$, and 0.052 , respectively, compared with those of the traditional MF method. The classification performance in different filters shows that the proposed MFPF can achieve the optimal accuracy for most kinds of specific classes. The optimal $\mathrm{OA}, \mathrm{AA}$, and $\mathrm{Ka}$ are obtained through the proposed MFPF. The visual performance presented in Figure 6 further demonstrates the conclusion of this experiment.

Table 1. Classification performance comparisons (\%) on the JX01 dataset using SVM and different image filters (training samples: 100 points/class).

\begin{tabular}{cccccc}
\hline Class & $\begin{array}{c}\text { Raw Image } \\
\text { without Filter }\end{array}$ & MF w=3×3 & MedF w=3×3 & $\begin{array}{c}\text { MMF [23] } \mathbf{T}_{\mathbf{1}}=\mathbf{1 5}, \\
\mathbf{T}_{\mathbf{2}}=\mathbf{1 0 0}\end{array}$ & $\begin{array}{c}\text { Proposed } \\
\mathbf{M F P F}\end{array}$ \\
\hline Road & 96.10 & $\mathbf{9 7 . 8 0}$ & 97.70 & 96.60 & 95.00 \\
Grass & 93.60 & 93.50 & 93.40 & 97.10 & $\mathbf{9 8 . 2 0}$ \\
Building & 99.30 & 99.50 & 99.50 & 99.50 & $\mathbf{9 9 . 5 0}$ \\
Shadow & $\mathbf{8 9 . 1 0}$ & 84.30 & 80.90 & 81.40 & 87.80 \\
Trees & 93.40 & $\mathbf{9 3 . 9 0}$ & 93.70 & 93.00 & 93.40 \\
Water & 95.00 & 96.20 & 93.90 & 98.60 & $\mathbf{9 9 . 0 0}$ \\
Soil & 57.00 & 56.90 & 57.00 & 57.30 & $\mathbf{7 0 . 4 0}$ \\
OA & 88.52 & 88.31 & 87.79 & 88.22 & $\mathbf{9 2 . 4 3}$ \\
AA & 89.08 & 88.89 & 88.01 & 89.07 & $\mathbf{9 1 . 8 8}$ \\
KA & 0.851 & 0.848 & 0.842 & 0.847 & $\mathbf{0 . 9 0 0}$ \\
\hline
\end{tabular}

Note: The bold texts in each row correspond to the optimal accuracies of the comparisons. 

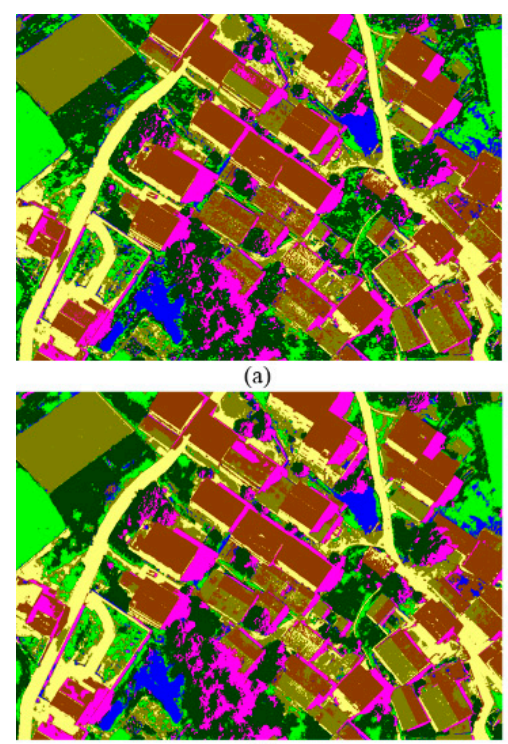

(d)

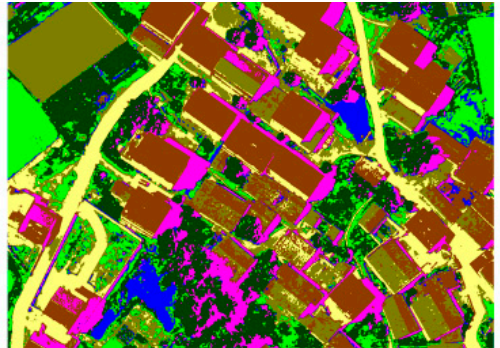

(b)

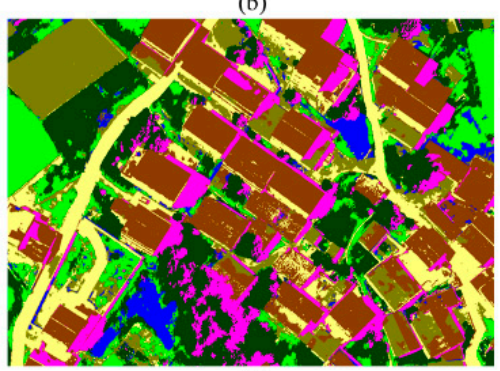

(e)

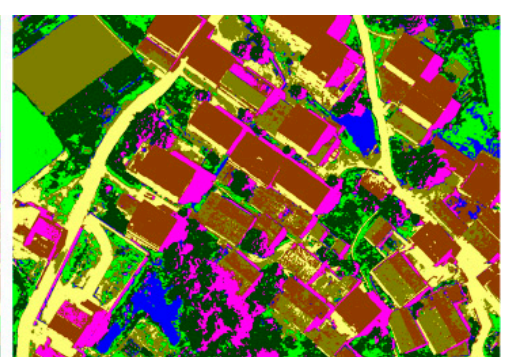

(c)

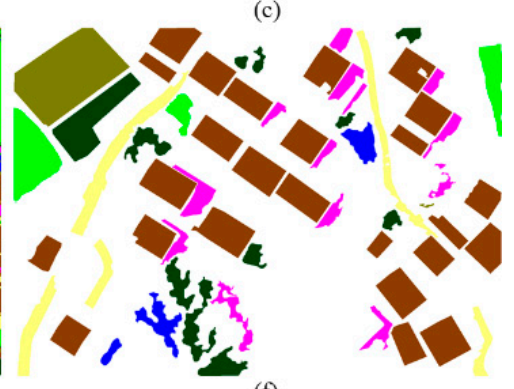

(f)

Figure 6. Classification maps of the JX01 dataset obtained using SVM with different filtered images: (a) raw image without filter; (b) MF with a $3 \times 3$ window; (c) MedF with a $3 \times 3$ window; (d) MMF with $T_{1}=15$ and $T_{2}=100 ;(\mathbf{e})$ proposed MFPF; (f) ground reference map.

The second experiment is conducted on the Pavia University dataset to demonstrate the effectiveness and adaptability of the proposed MFPF further. The experimental results are presented as follows. (1) The proposed MFPF achieves the optimal accuracies in terms of OA, AA, and Ka in the Pavia University image in comparison with traditional filters, such as the MF, MedF, and previous MMF. Table 2 presents that the OA and AA of the proposed MFPF are $94.18 \%$ and $94.14 \%$ for the KNN classifier, correspondingly, and those of MMF are $83.92 \%$ and $81.38 \%$, respectively. Improvements of $10.26 \%$ and $12.76 \%$ in terms of OA and AA, respectively, are obtained by the proposed MFPF compared with those of MMF. The comparison details for the KNN are summarized in Table 3 to demonstrate the superiority of the proposed MFPF. (2) The results based on the different classifiers and filters imply that the proposed MFPF is effective and robust for the SVM, KNN, and RT classifiers. The proposed MFPF performs the optimal accuracies for each classifier in comparison with those of the raw image without any processing and the images filtered with MF, MedF, and MMF.

Table 2. Evaluation of the performance of the proposed MFPF on the Pavia University image in terms of different classifiers ("S" indicates the number of training samples).

\begin{tabular}{|c|c|c|c|c|c|c|}
\hline Classifier & & $\begin{array}{c}\text { Raw Image } \\
\text { without } \\
\text { Filter }\end{array}$ & $\mathbf{M F} \mathbf{w}=3 \times 3$ & MedF w $=9 \times 9$ & $\begin{array}{c}\text { MMF [23] } \\
T_{1}=25 \\
T_{2}=100\end{array}$ & $\begin{array}{c}\text { Proposed } \\
\text { MFPF }\end{array}$ \\
\hline \multirow{4}{*}{$\operatorname{SVM}(S=130)$} & OA & 63.04 & 72.03 & 74.00 & 75.50 & 83.57 \\
\hline & AA & 64.99 & 72.33 & 76.05 & 77.14 & 83.25 \\
\hline & $\mathrm{Ka}$ & 0.539 & 0.641 & 0.666 & 0.684 & 0.775 \\
\hline & OA & 66.91 & 73.60 & 80.30 & 83.92 & 94.18 \\
\hline \multirow[t]{2}{*}{$\mathrm{KNN}(\mathrm{S}=500)$} & AA & 67.69 & 71.47 & 77.27 & 81.38 & 94.14 \\
\hline & $\mathrm{Ka}$ & 0.588 & 0.667 & 0.750 & 0.793 & 0.924 \\
\hline \multirow{3}{*}{$\mathrm{RT}(\mathrm{S}=450)$} & OA & 65.64 & 71.68 & 78.96 & 79.13 & 92.36 \\
\hline & AA & 66.48 & 72.01 & 77.00 & 77.50 & 92.59 \\
\hline & $\mathrm{Ka}$ & 0.573 & 0.645 & 0.733 & 0.734 & 0.901 \\
\hline
\end{tabular}

Note: The bold texts in each row correspond to the optimal accuracies of the comparisons. 
Table 3. Class-specific user accuracy (\%) of the Pavia University image for the different methods with KNN.

\begin{tabular}{cccccc}
\hline Class & $\begin{array}{c}\text { Raw Image } \\
\text { without Filter }\end{array}$ & MF (w=3×3) & MedF (w=9×9) & $\begin{array}{c}\text { MMF [23] } \\
\mathbf{T}_{\mathbf{1}}=\mathbf{2 5}, \\
\mathbf{T}_{\mathbf{2}}=\mathbf{1 0 0}\end{array}$ & $\begin{array}{c}\text { Proposed } \\
\mathbf{M F P F}\end{array}$ \\
\hline Asphalt & 87.80 & 94.40 & 96.30 & 96.10 & $\mathbf{9 7 . 3 0}$ \\
Meadows & 86.30 & 90.00 & 93.70 & 94.40 & $\mathbf{9 9 . 3 0}$ \\
Gravel & 42.80 & 48.00 & 66.20 & 71.80 & $\mathbf{9 0 . 0 0}$ \\
Tress & 55.90 & 59.70 & 58.90 & 67.90 & $\mathbf{9 2 . 3 0}$ \\
Painted & 82.60 & 73.70 & 82.40 & 85.00 & $\mathbf{9 7 . 4 0}$ \\
Bare soil & 34.10 & 44.80 & 54.80 & 59.70 & $\mathbf{7 5 . 6 0}$ \\
Bitumen & 55.20 & 57.70 & 71.60 & 79.90 & $\mathbf{9 9 . 4 0}$ \\
Self-blocking & 72.10 & 77.70 & 89.90 & 87.50 & $\mathbf{9 8 . 8 0}$ \\
bricks & 92.50 & 97.20 & 81.60 & 90.20 & $\mathbf{9 7 . 3 0}$ \\
Shadows & 66.91 & 73.60 & 80.30 & 83.92 & $\mathbf{9 4 . 1 8}$ \\
OA & 67.69 & 71.47 & 77.27 & 81.38 & $\mathbf{9 4 . 1 4}$ \\
AA & 0.588 & 0.667 & 0.750 & 0.793 & $\mathbf{0 . 9 2 4}$ \\
KA &
\end{tabular}

Note: The bold texts in each row correspond to the optimal accuracies of the comparisons.

Three demonstrations with the specific KNN, SVM, and RT classifier are respectively illustrated for the corresponding visual performance in Figures 7-9. The visual comparison and observation results clearly demonstrate that the classification map obtained through the proposed MFPF performs with minimal noise, and the intra-class homogeneity, such as the meadow highlighted by the rectangle in Figures 7-9, is improved. The boundary of ground targets can be preserved well in the classification map obtained through the proposed MFPF.

In the third experiments on the ZH-6 dataset, the proposed MFPF is compared with the state-of-the-art spatial-spectral feature-based methods. Table 4 summarizes the results of the quantitative comparison. The comparison results show that the spatial-spectral features can improve the classification accuracies in comparison with those of the raw image without any processing. The improvement is approximately $5 \%$. However, the proposed MFPF not only achieves the optimal accuracy for most kinds of specific class but also obtains the highest accuracies in terms of OA, AA, and Ka in comparison with the state-of-the-art spatial-spectral feature-based methods. The visual performance depicted in Figure 10 supports the quantitative conclusion from this experiment.

Table 4. Class-specific user accuracy (\%) of the ZH-6 image for the different methods with SVM.

\begin{tabular}{ccccccc}
\hline Class & $\begin{array}{c}\text { Raw Image } \\
\text { without } \\
\text { Filter }\end{array}$ & EPFs [26] & RFs [27] & M_EMPS [25] & RGF [28] & $\begin{array}{c}\text { Proposed } \\
\text { MFPF }\end{array}$ \\
\hline Building & 87.00 & 89.30 & 90.30 & 68.30 & 91.90 & $\mathbf{9 3 . 4 0}$ \\
Road & 77.40 & 77.80 & 79.80 & 75.10 & $\mathbf{8 1 . 2 0}$ & 80.10 \\
Trees & 84.40 & 87.10 & 86.00 & 84.40 & 89.90 & $\mathbf{9 0 . 9 0}$ \\
Grass & 73.60 & 75.10 & 78.90 & 67.50 & 79.60 & $\mathbf{8 1 . 8 0}$ \\
Water & 4.90 & 5.61 & 5.61 & 5.87 & 6.22 & $\mathbf{1 1 . 0 0}$ \\
Bare soil & 6.18 & 6.64 & 8.38 & 7.90 & 7.92 & $\mathbf{1 0 . 2 0}$ \\
Pools & 32.9 & 33.90 & 30.70 & $\mathbf{3 5 . 2 0}$ & 35.10 & 34.80 \\
OA & 67.21 & 69.11 & 70.41 & 65.20 & 71.90 & $\mathbf{7 4 . 9 6}$ \\
AA & 52.35 & 53.64 & 54.26 & 49.19 & 55.98 & $\mathbf{5 7 . 4 5}$ \\
Ka & 0.579 & 0.601 & 0.618 & 0.550 & 0.638 & $\mathbf{0 . 6 7 4}$ \\
\hline
\end{tabular}

Note: The bold texts in each row correspond to the optimal accuracies of the comparisons. 


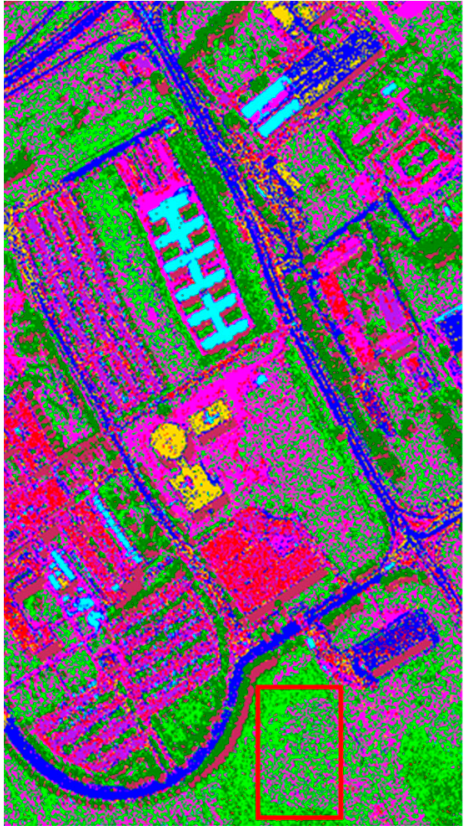

(a)

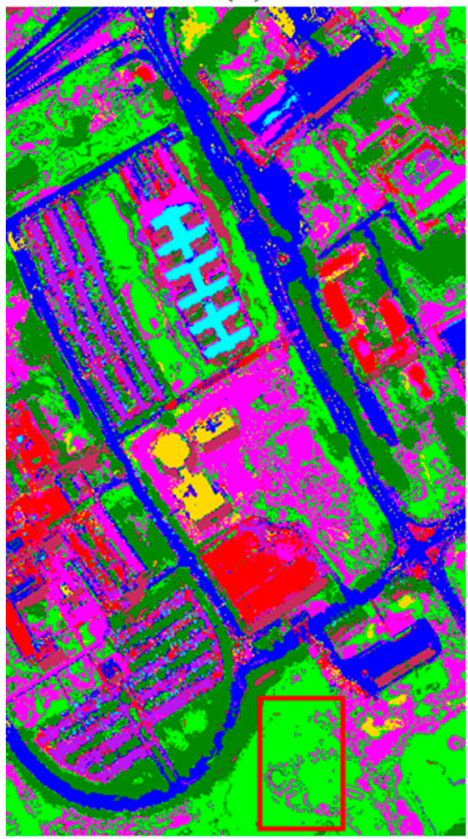

(d)

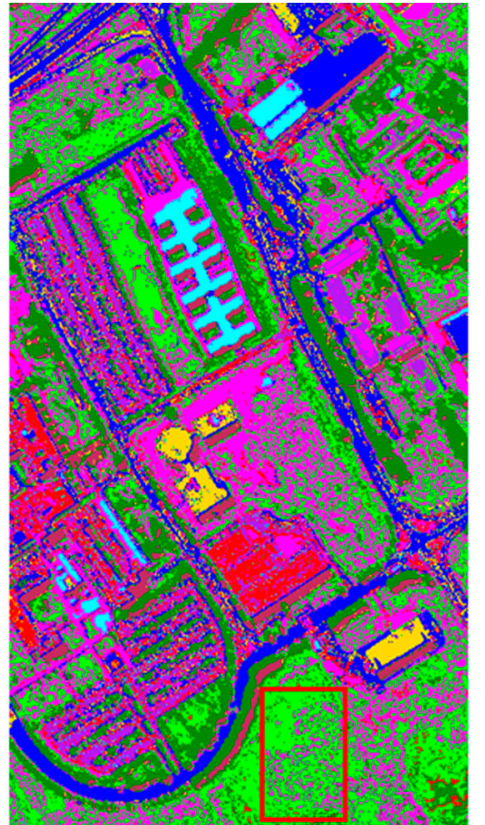

(b)

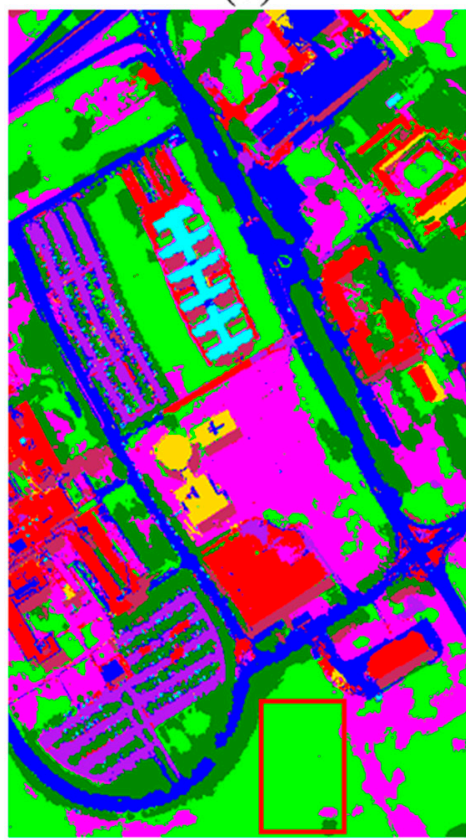

(e)

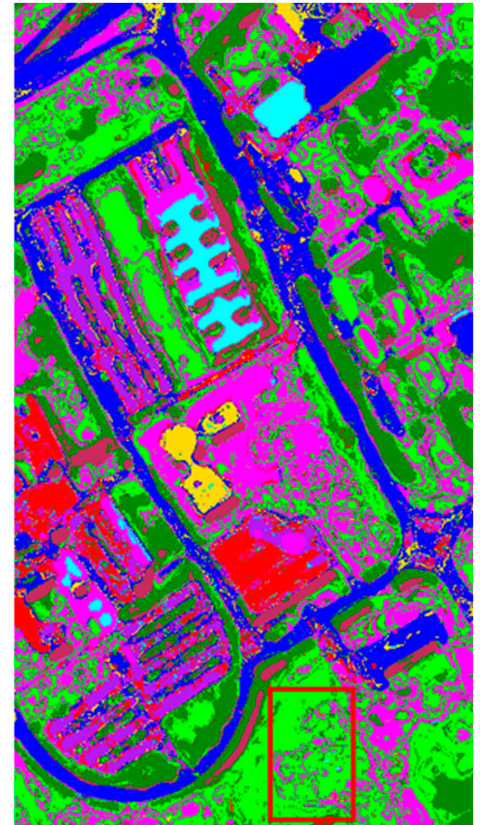

(c)

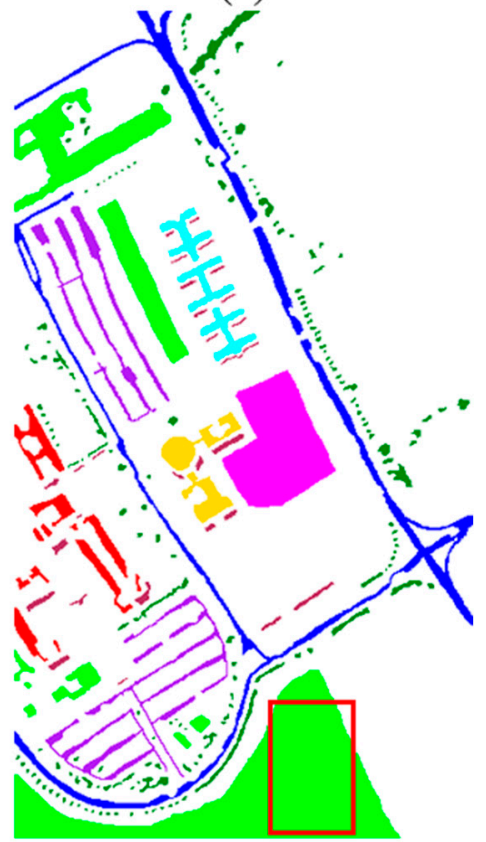

(f)

Figure 7. Classification maps of the Pavia University dataset obtained through KNN with different filtered images: (a) raw image without filter; (b) MF with a $3 \times 3$ window; (c) MedF with a $9 \times 9$ window; (d) MMF with $T_{1}=25$ and $T_{2}=100 ;(\mathbf{e})$ proposed MFPF; (f) ground reference map. 


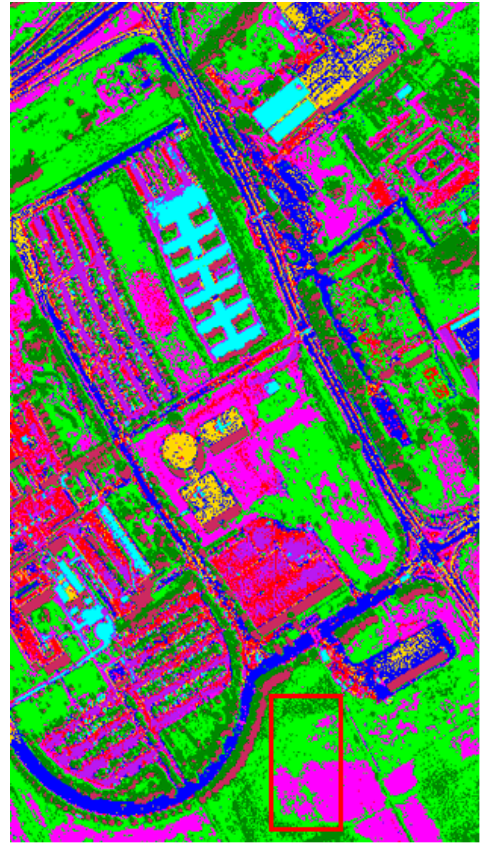

(a)

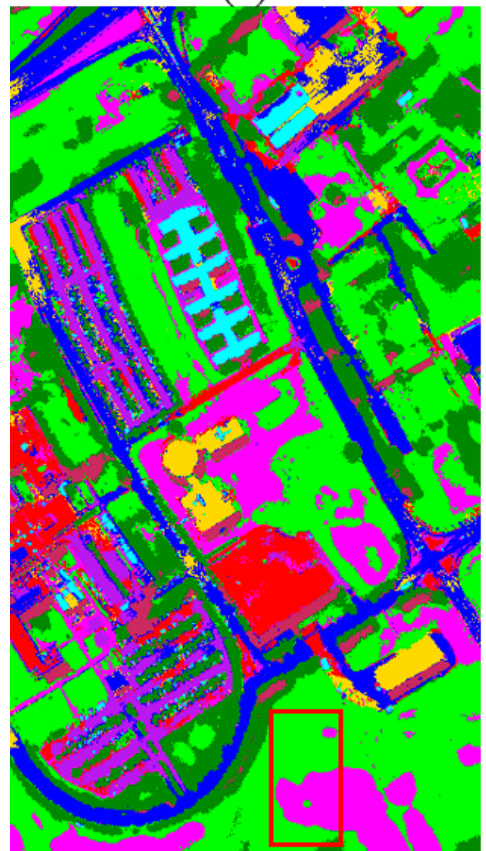

(d)

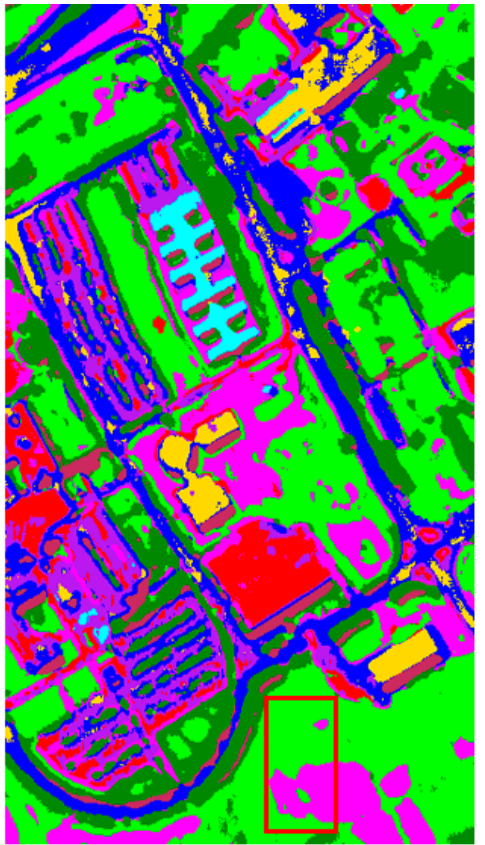

(b)

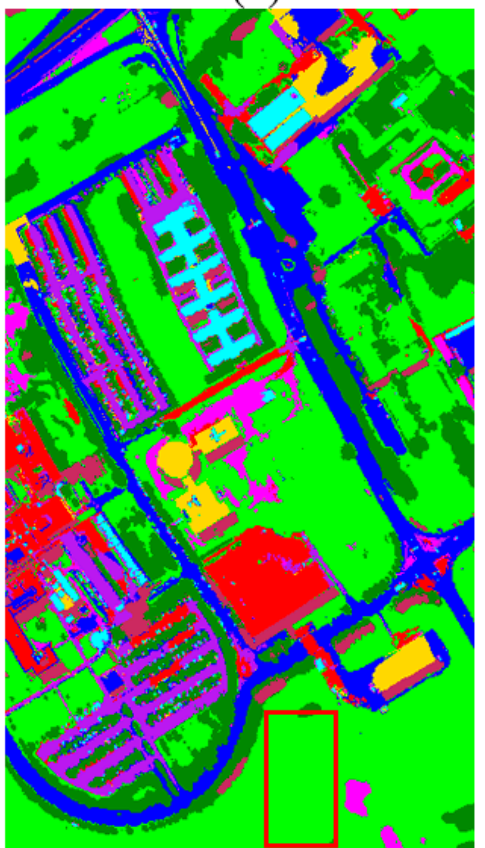

(e)

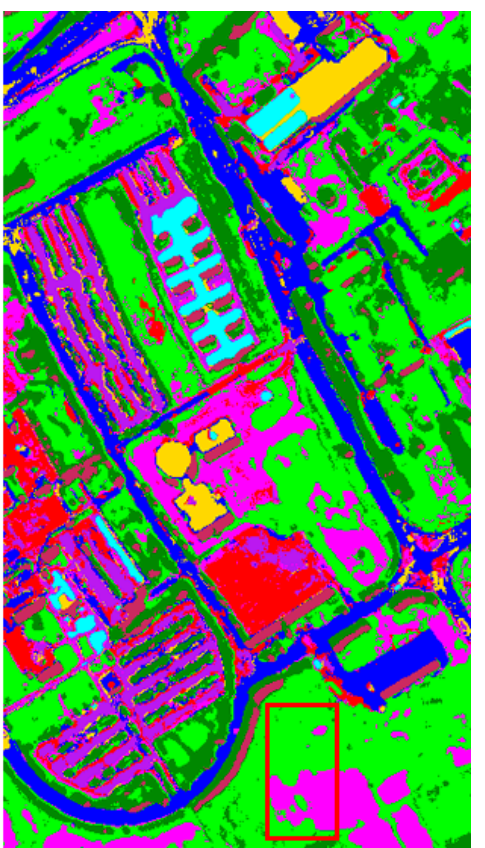

(c)

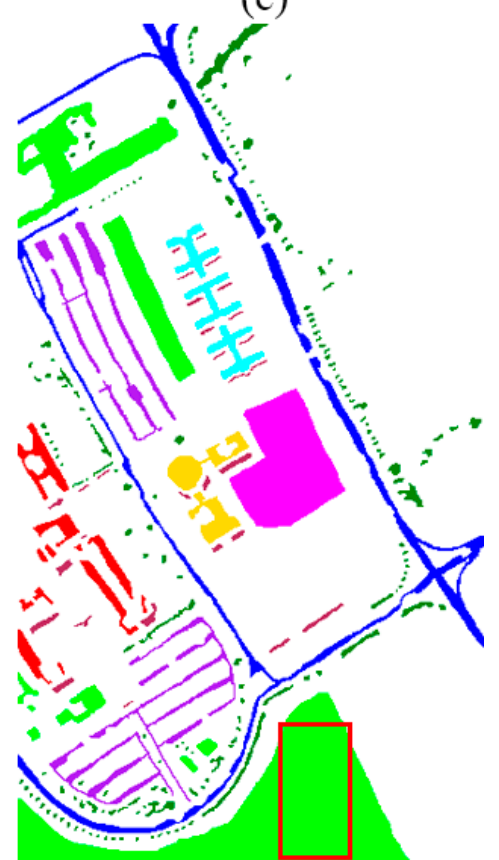

(f)

Figure 8. Classification maps of the Pavia University dataset obtained through SVM with different filtered images: (a) raw image without filter; (b) MF with a $7 \times 7$ window; (c) MedF with a $5 \times 5$ window; (d) MMF with $T_{1}=30$ and $T_{2}=100$; (e) proposed MFPF; (f) ground reference map. 


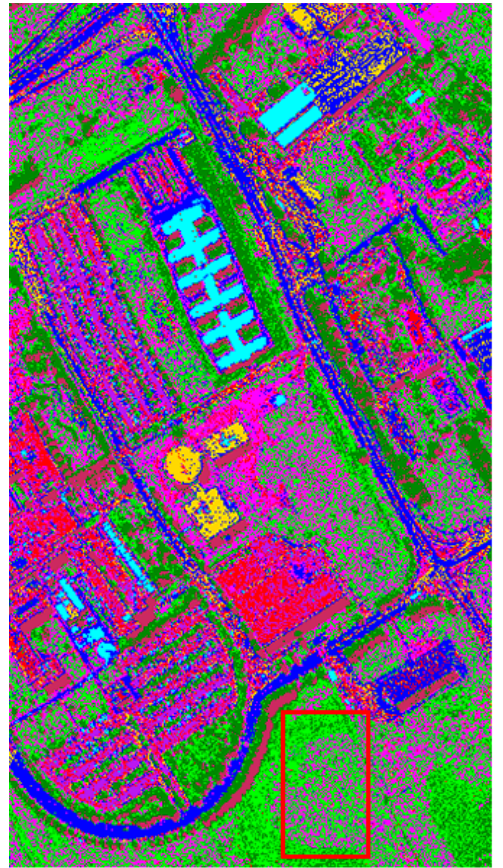

(a)

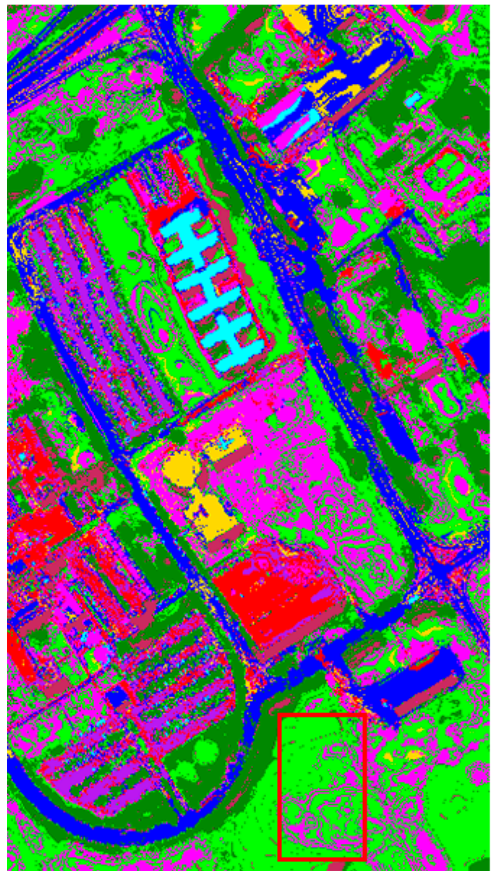

(d)

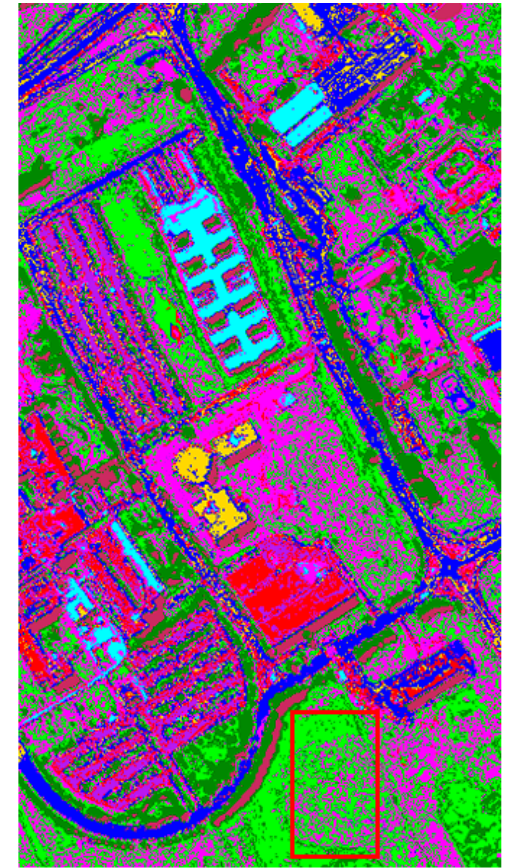

(b)

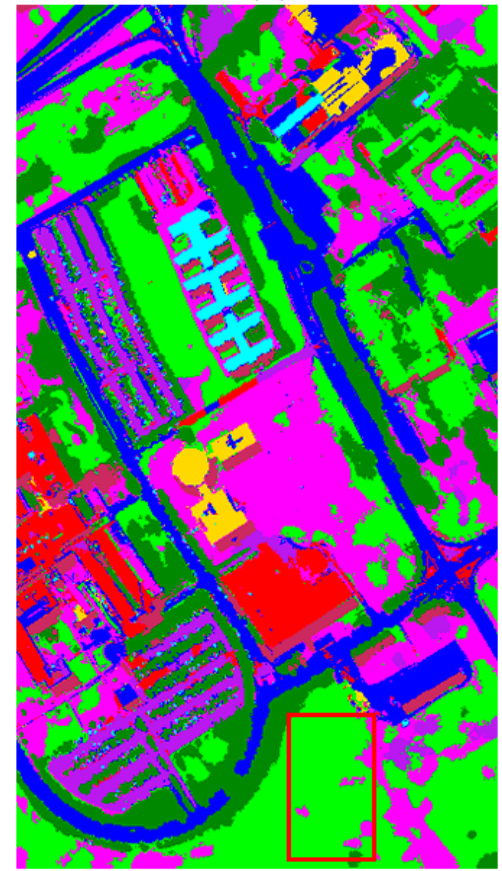

(e)

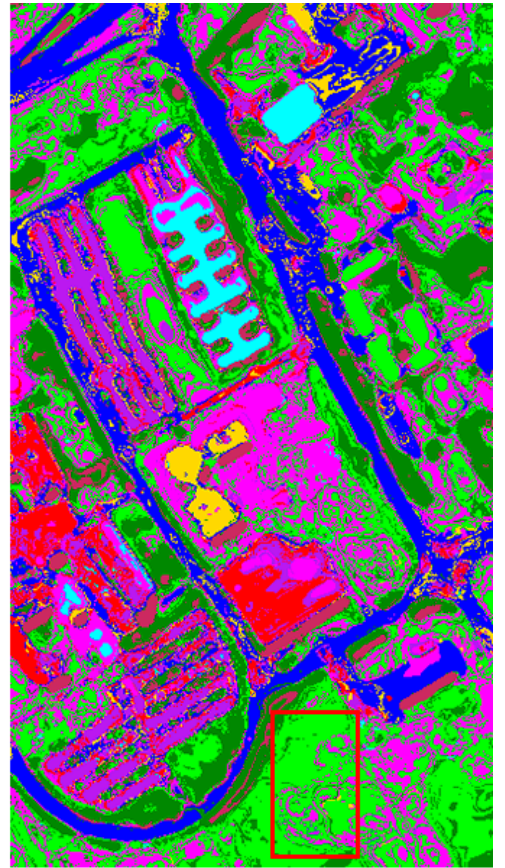

(c)

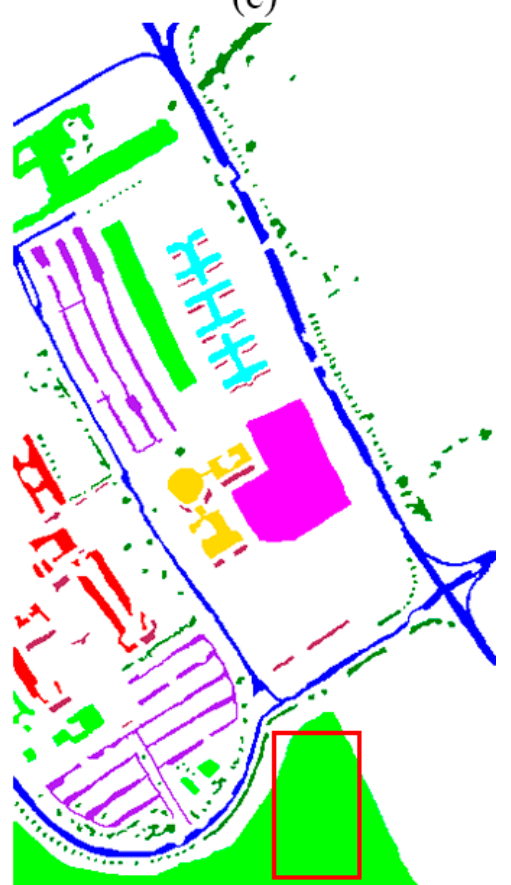

(f)

Figure 9. Classification maps of the Pavia University dataset obtained through RT with different filtered images: (a) raw image without filter; (b) MF with a $3 \times 3$ window; (c) MedF with a $9 \times 9$ window; (d) MMF with $T_{1}=30$ and $T_{2}=100 ;(\mathbf{e})$ proposed MFPF; (f) ground reference map. 


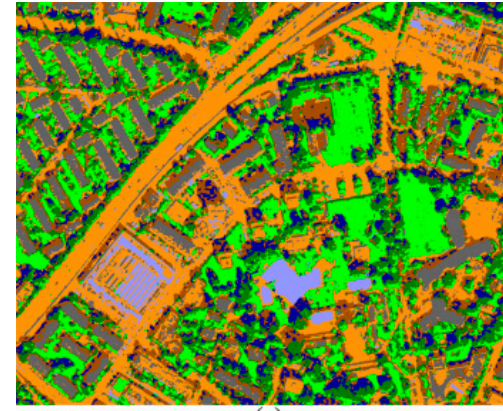

(a)

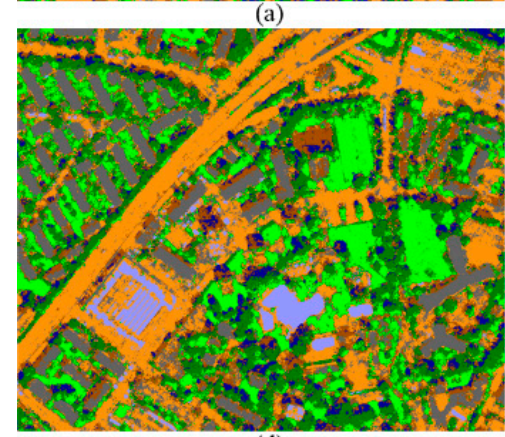

(d)

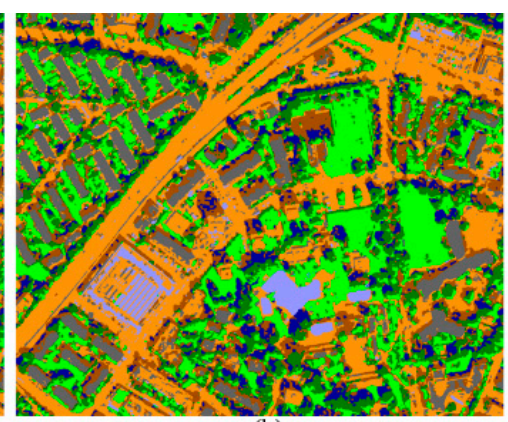

(b)

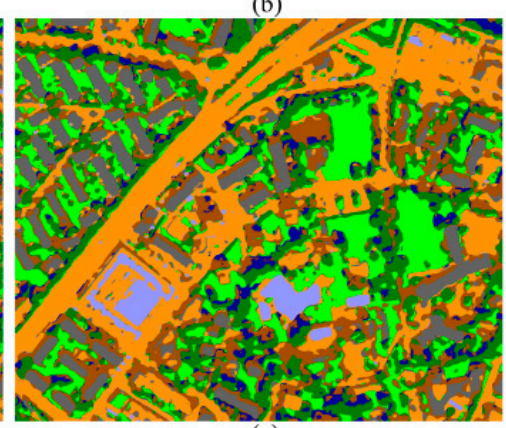

(e)

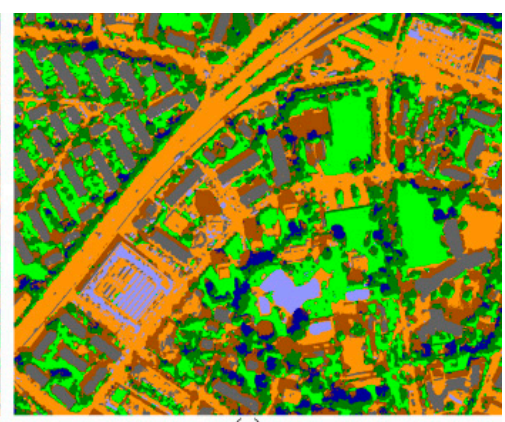

(c)

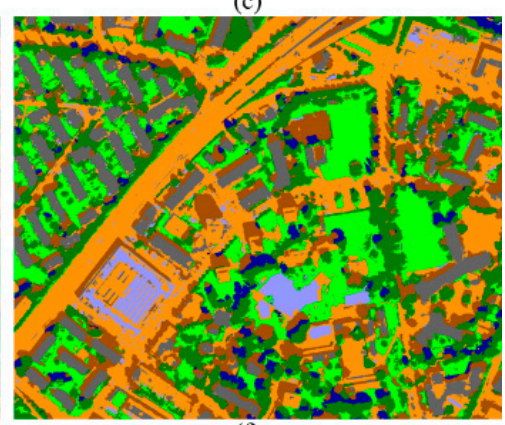

(f)

Figure 10. Classification maps of the ZH-6 dataset obtained through SVM with spatial-spectral feature images and the proposed methods: (a) raw image without filter; (b) EPFs [23]; (c) RFs [24]; (d) M_EMPs [22]; (e) RGF [25]; (f) proposed MFPF.

The experimental results based on the three real VHR remote sensing images show that the following aspects of the proposed MFPF can be achieved. First, in the proposed approach, a novel image filter, is effective in smoothing the noise of VHR remote sensing images. The proposed MFP-based approach called MFPF performs better than the MF, MedF, and the previous MMF in terms of accuracies in land cover classification of VHR remote sensing images. Second, the proposed MFPF is robust and effective for classical classifiers, namely, SVM, KNN, and RT. Finally, the proposed MFPF has a certain superiority to the spatial-spectral feature-based methods in comparison with the state-of-the-art VHR image classification methods; for example, the improvements are $3.06 \%-9.76 \%$ and $1.47 \%-8.26 \%$, in terms of $\mathrm{OA}$ and $\mathrm{AA}$, respectively.

\section{Discussion}

The relationship between the accuracies and the number of training samples for the different methods is analyzed in this section. This task is initiated to promote the potential application of the proposed MFPF. As shown in Figures 11-13, the accuracies of each approach are improved with an increase in the number of training samples. For example, in Figure 11a-c, the OAs of the JX01 dataset and SVM classifier increase from $79.37 \%$ to $87.75 \%$, whereas the number of training samples for the proposed MFPF increases from 5 points/class to 10 points/class. The OA of the proposed approach gradually increases to $93.43 \%$ when the number of training samples reaches 100 points/class. The fluctuation in the method accuracies may be due to the uncertain spatial distribution of the training samples for each test. 


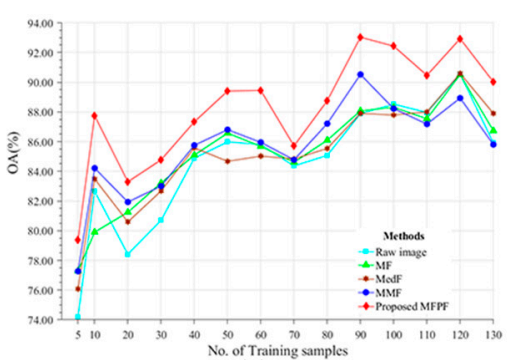

(a)

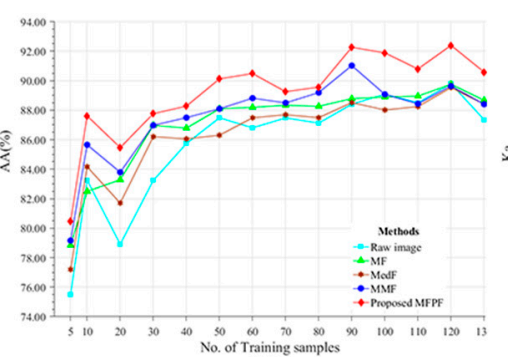

(b)

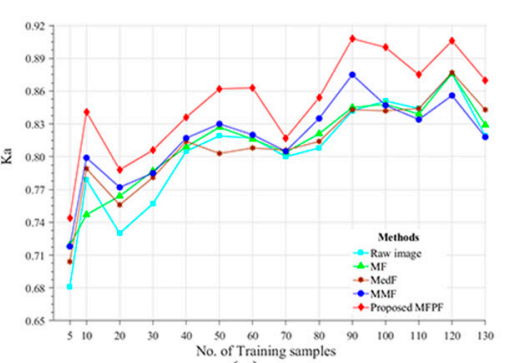

(c)

Figure 11. Relationship between the classification maps and the number of training samples for the different methods: $(\mathbf{a}-\mathbf{c})$ relationship between accuracies and number of training samples for the JX01 dataset using SVM.

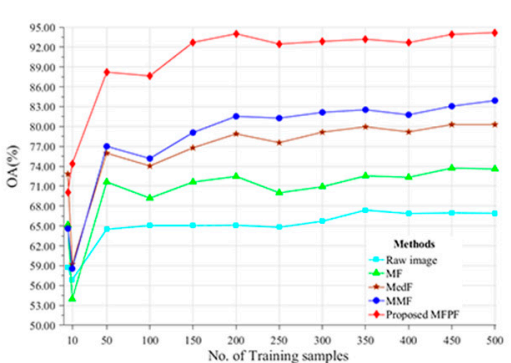

(a)

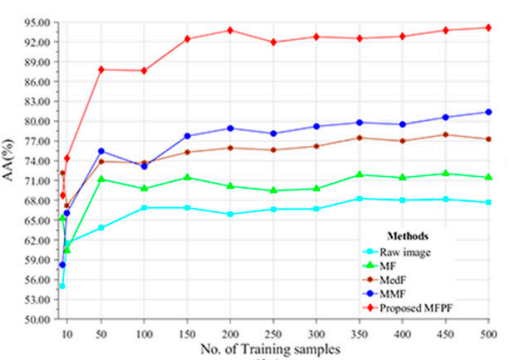

(b)

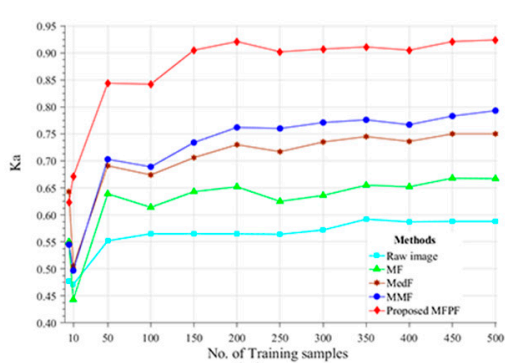

(c)

Figure 12. Relationship between the classification maps and the number of training samples for the different methods: $(\mathbf{a}-\mathbf{c})$ relationship between accuracies and number of training samples for the Pavia University dataset using KNN.

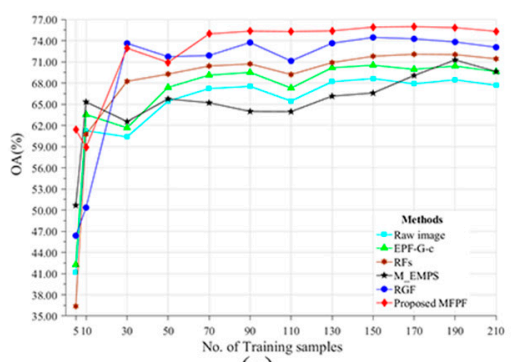

(a)

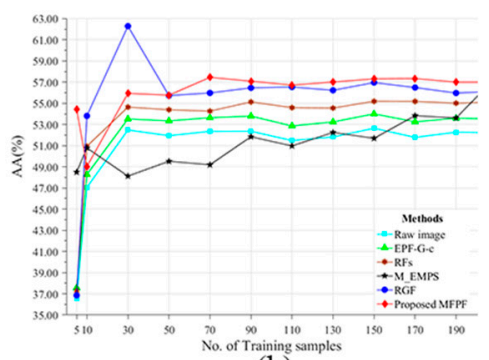

(b)

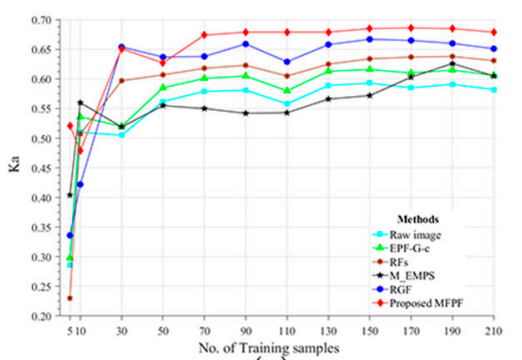

(c)

Figure 13. Relationship between the classification maps and the number of training samples for the different methods: $(\mathbf{a}-\mathbf{c})$ relationship between accuracies and number of training samples for the ZH-6 dataset using SVM.

The accuracies on the Pavia University image and ZH-6 dataset with KNN and SVM classifier also swiftly increase when the number of training samples increases from 5 points/class to 10 points/class. However, the sensitivity between the accuracies and the number of training samples stabilizes when the latter is more than 50 points/class. The OA of the proposed MFPF only increases from $88.21 \%$ to $94.18 \%$. By contrast, the number of training samples increases from 50 points/class to 500 points/class for the KNN classifier, as illustrated in Figure 12a-c.

The above observation and the comparison results for the three datasets clearly demonstrate that the proposed MFPF achieves enhanced accuracies under an equal level of the training sample for each filter. The accuracies of the proposed MFPF will increase and stabilize. This finding is helpful for the number of training samples selected for the proposed MFPF. 


\section{Conclusions}

In the present work, a simple but powerful approach called MFPF was proposed for VHR remote sensing image classification. Instead of using the filter with a single processing scale, filter profiles were built in the proposed MFPF approach by filtering the image with a series of different parameters. The proposed MFPF comprises the following three major steps. (1) MFPs were first constructed through a modified mean filter with different scale parameters. (2) The classical PCA was then adopted for reducing the dimension of MPFs, and the top three components were taken as the input feature for a supervised classifier to obtain the initial classification map. (3) Finally, the adaptive spatial region of each filter was adopted for post-processing the initial classification map. The contributions and advantages of the proposed MFPF are summarized as follows.

(1) The proposed MFPF provided competitive accuracies in land cover classification of VHR remote sensing images. As an extension of MMF [20], the proposed MFPF achieved the best accuracy compared with that of the MMF, MF, MedF, and the raw image without any filter processing Furthermore, the results of the second experiment indicated that the proposed MFPF is more robust for the different classifiers compared with that of the MF, MedF, and MMF. In addition, the classification results achieved by the proposed MFPF clearly demonstrated its effectiveness and superiority in terms of visual performance and quantitative accuracies compared with those based on the classical spatial-spectral feature extraction approaches, including EPFs [26], RFs [27], M_EMPs [25], and RGF [28].

(2) To the best of the author's knowledge, this study is the first to promote the idea of multi-scale filter profile construction to improve the classification performance with VHR remote sensing images. Experimental results indicate that the proposed MFPF can smooth the salt-and-pepper noise of classification maps because this approach can cover various targets with different shapes and sizes. Furthermore, the post-processing strategy with the adaptive region of each filter in the proposed MFPF can further smooth the noise in the initial classification map and improve its performance and accuracy.

The experimental results based on three real VHR remote sensing images acquired using different sensors and platforms indicated that the proposed MFPF is effective for smoothing the noise of classification maps and improving classification accuracies. An advantage of the proposed MFP is its ability to improve the intra-class spectral homogeneity while preserving the edge of targets. This improvement helps smooth the noise of the classification map and upgrades the recognition accuracies. Another major advantage of the proposed MFPF is its capability to avoid hard-tuning of parameters for a pre-given VHR image, which will be relatively useful in real applications.

Overall, the newly proposed approach is a promising framework for VHR remote sensing image classification. In future works, the robustness and adaptability of the proposed approach will be investigated on additional kinds of remote sensing images, such as the WorldView 4.0 VHR sensing images.

Author Contributions: Conceptualization, Z.L. and G.L.; methodology, Z.L.; software, G.L.; validation, Y.C.; formal analysis, J.A.B.; data curation, G.L. Writing-Original Draft preparation, Z.L., and Y.C.; Writing-Review and Editing, J.A.B.; visualization, G.L.; supervision, Z.L.; project administration, Z.L. and Y.C.; funding acquisition, Y.C.

Funding: This research was funded by the National Science Foundation China (61701396 and 41501378) and the Natural Science Foundation of Shaan Xi Province (2018JQ4009).

Acknowledgments: The authors would like to express their gratitude to the editor-in-chief, the associate editor, and the reviewers for their insightful comments and suggestions.

Conflicts of Interest: The authors declare no conflict of interest. 


\section{References}

1. Wang, Q.; Lin, J.; Yuan, Y. Salient band selection for hyperspectral image classification via manifold ranking. IEEE Trans. Neural Netw. Learn. Syst. 2016, 27, 1279-1289. [CrossRef] [PubMed]

2. Kabisch, N.; Selsam, P.; Kirsten, T.; Lausch, A.; Bumberger, J. A multi-sensor and multi-temporal remote sensing approach to detect land cover change dynamics in heterogeneous urban landscapes. Ecol. Indic. 2019, 99, 273-282. [CrossRef]

3. Li, J.; Huang, X.; Hu, T.; Jia, X.; Benediktsson, J.A. A novel unsupervised sample collection method for urban land-cover mapping using landsat imagery. IEEE Trans. Geosci. Remote Sens. 2019, 57, 3933-3951. [CrossRef]

4. Wang, Q.; Gao, J.; Li, X. Weakly supervised adversarial domain adaptation for semantic segmentation in urban scenes. IEEE Trans. Image Process. 2019. [CrossRef] [PubMed]

5. Ding, B.; Wen, G.; Huang, X.; Ma, C.; Yang, X. Data augmentation by multilevel reconstruction using attributed scattering center for sar target recognition. IEEE Geosci. Remote Sens. Lett. 2017, 14, 979-983. [CrossRef]

6. Zhang, L.; Zhang, Y. Airport detection and aircraft recognition based on two-layer saliency model in high spatial resolution remote-sensing images. IEEE J. Sel. Top. Appl. Earth Obs. Remote Sens. 2016, 10, 1511-1524. [CrossRef]

7. Wang, Q.; Wan, J.; Nie, F.; Liu, B.; Yan, C.; Li, X. Hierarchical feature selection for random projection. IEEE Trans. Neural Netw. Learn. Syst. 2018, 30, 1581-1586. [CrossRef]

8. Lv, P.; Zhong, Y.; Zhao, J.; Ma, A.; Zhang, L. Change detection based on structural conditional random field framework for high spatial resolution remote sensing imagery. In Proceedings of the 2017 IEEE International Geoscience and Remote Sensing Symposium (IGARSS), Fort Worth, TX, USA, 23-28 July 2017; pp. 1059-1062.

9. Xu, L.; Jing, W.; Song, H.; Chen, G. High-resolution remote sensing image change detection combined with pixel-level and object-level. IEEE Access 2019, 7, 78909-78918. [CrossRef]

10. Gianinetto, M.; Rusmini, M.; Candiani, G.; Via, G.D.; Frassy, F.; Maianti, P.; Marchesi, A.; Nodari, F.R.; Dini, L. Hierarchical classification of complex landscape with vhr pan-sharpened satellite data and obia techniques. Eur. J. Remote Sens. 2014, 47, 229-250. [CrossRef]

11. Lv, Z.; He, H.; Benediktsson, J.; Huang, H. A generalized image scene decomposition-based system for supervised classification of very high resolution remote sensing imagery. Remote Sens. 2016, 8, 814. [CrossRef]

12. Huang, X.; Zhang, L. An svm ensemble approach combining spectral, structural, and semantic features for the classification of high-resolution remotely sensed imagery. IEEE Trans. Geosci. Remote Sens. 2012, 51, 257-272. [CrossRef]

13. Huang, X.; Lu, Q.; Zhang, L. A multi-index learning approach for classification of high-resolution remotely sensed images over urban areas. ISPRS J. Photogramm. Remote Sens. 2014, 90, 36-48. [CrossRef]

14. Demir, B.; Bruzzone, L. Histogram-based attribute profiles for classification of very high resolution remote sensing images. IEEE Trans. Geosci. Remote Sens. 2015, 54, 2096-2107. [CrossRef]

15. Cheng, G.; Han, J.; Guo, L.; Liu, Z.; Bu, S.; Ren, J. Effective and efficient midlevel visual elements-oriented land-use classification using vhr remote sensing images. IEEE Trans. Geosci. Remote Sens. 2015, 53, 4238-4249. [CrossRef]

16. Zhao, W.; Du, S.; Wang, Q.; Emery, W.J. Contextually guided very-high-resolution imagery classification with semantic segments. ISPRS J. Photogramm. Remote Sens. 2017, 132, 48-60. [CrossRef]

17. Liu, L.; Jia, Z.; Yang, J.; Kasabov, N. A remote sensing image enhancement method using mean filter and unsharp masking in non-subsampled contourlet transform domain. Trans. Inst. Meas. Control 2017, 39, 183-193. [CrossRef]

18. Huang, W.; Wang, R.; Gong, X.; Chen, Y. Iterative deblending of simultaneous-source seismic data with structuring median constraint. IEEE Geosci. Remote Sens. Lett. 2017, 15, 58-62. [CrossRef]

19. Zhang, P.; Li, F. A new adaptive weighted mean filter for removing salt-and-pepper noise. IEEE Signal Process. Lett. 2014, 21, 1280-1283. [CrossRef]

20. Lin, T.; Zhang, Y.; Müller-Petke, M. Random noise suppression of magnetic resonance sounding oscillating signal by combining empirical mode decomposition and time-frequency peak filtering. IEEE Access 2019, 7, 79917-79926. [CrossRef]

21. Soille, P. Morphological Image Analysis: Principles and Applications; Springer Science \& Business Media: Berlin, Germany, 2013.

22. He, L.; Li, J.; Plaza, A.; Li, Y. Discriminative low-rank gabor filtering for spectral-spatial hyperspectral image classification. IEEE Trans. Geosci. Remote Sens. 2016, 55, 1381-1395. [CrossRef] 
23. ZhiYong, L.; Shi, W.; Benediktsson, J.A.; Gao, L. A modified mean filter for improving the classification performance of very high-resolution remote-sensing imagery. Int. J. Remote Sens. 2018, 39, 770-785. [CrossRef]

24. Dalla Mura, M.; Benediktsson, J.A.; Waske, B.; Bruzzone, L. Morphological attribute profiles for the analysis of very high resolution images. IEEE Trans. Geosci. Remote Sens. 2010, 48, 3747-3762. [CrossRef]

25. Lv, Z.Y.; Zhang, P.; Benediktsson, J.A.; Shi, W.Z. Morphological profiles based on differently shaped structuring elements for classification of images with very high spatial resolution. IEEE J. Sel. Top. Appl. Earth Obs. Remote Sens. 2014, 7, 4644-4652. [CrossRef]

26. Kang, X.; Li, S.; Benediktsson, J.A. Spectral-spatial hyperspectral image classification with edge-preserving filtering. IEEE Trans. Geosci. Remote Sens. 2013, 52, 2666-2677. [CrossRef]

27. Kang, X.; Li, S.; Benediktsson, J.A. Feature extraction of hyperspectral images with image fusion and recursive filtering. IEEE Trans. Geosci. Remote Sens. 2013, 52, 3742-3752. [CrossRef]

28. Zhang, Q.; Shen, X.; Xu, L.; Jia, J. Rolling Guidance Filter; European Conference on Computer Vision; Springer: Cham, Switzerland, 2014; pp. 815-830.

29. Li, M.; Zang, S.; Zhang, B.; Li, S.; Wu, C. A review of remote sensing image classification techniques: The role of spatio-contextual information. Eur. J. Remote Sens. 2014, 47, 389-411. [CrossRef]

30. Li, C.; Yang, S.; Yang, Y.; Gao, H.; Zhao, J.; Qu, X.; Wang, Y.; Yao, D.; Gao, J. Hyperspectral remote sensing image classification based on maximum overlap pooling convolutional neural network. Sensors 2018, 18, 3587. [CrossRef] [PubMed]

31. Shi, C.; Pun, C.-M. Adaptive multi-scale deep neural networks with perceptual loss for panchromatic and multispectral images classification. Inf. Sci. 2019, 490,1-17. [CrossRef]

32. Chen, Y.; Jiang, H.; Li, C.; Jia, X.; Ghamisi, P. Deep feature extraction and classification of hyperspectral images based on convolutional neural networks. IEEE Trans. Geosci. Remote Sens. 2016, 54, 6232-6251. [CrossRef]

33. Wilkinson, G.G. Results and implications of a study of fifteen years of satellite image classification experiments. IEEE Trans. Geosci. Remote Sens. 2005, 43, 433-440. [CrossRef]

34. Liu, D.; Xia, F. Assessing object-based classification: Advantages and limitations. Remote Sens. Lett. 2010, 1, 187-194. [CrossRef]

35. Tang, Y.; Atkinson, P.M.; Wardrop, N.A.; Zhang, J. Multiple-point geostatistical simulation for post-processing a remotely sensed land cover classification. Spat. Stat. 2013, 5, 69-84. [CrossRef]

36. Huang, X.; Lu, Q.; Zhang, L.; Plaza, A. New postprocessing methods for remote sensing image classification: A systematic study. IEEE Trans. Geosci. Remote Sens. 2014, 52, 7140-7159. [CrossRef]

37. Lv, Z.; Zhang, X.; Benediktsson, J.A. Developing a general post-classification framework for land-cover mapping improvement using high-spatial-resolution remote sensing imagery. Remote Sens. Lett. 2017, 8, 607-616. [CrossRef]

38. Lv, Z.; Liu, T.; Wan, Y.; Benediktsson, J.A.; Zhang, X. Post-processing approach for refining raw land cover change detection of very high-resolution remote sensing images. Remote Sens. 2018, 10, 472. [CrossRef]

39. Bouwmans, T.; Sobral, A.; Javed, S.; Jung, S.K.; Zahzah, E.-H. Decomposition into low-rank plus additive matrices for background/foreground separation: A review for a comparative evaluation with a large-scale dataset. Comput. Sci. Rev. 2017, 23,1-71. [CrossRef]

40. Jolliffe, I.T.; Cadima, J. Principal component analysis: A review and recent developments. Philos. Trans. R. Soc. A Math. Phys. Eng. Sci. 2016, 374, 20150202. [CrossRef] [PubMed]

41. Tzeng, D.Y.; Berns, R.S. A review of principal component analysis and its applications to color technology. Color Res. Appl. 2005, 30, 84-98. [CrossRef]

42. Li, S.; Hao, Q.; Gao, G.; Kang, X. The effect of ground truth on performance evaluation of hyperspectral image classification. IEEE Trans. Geosci. Remote Sens. 2018, 56, 7195-7206. [CrossRef]

(C) 2019 by the authors. Licensee MDPI, Basel, Switzerland. This article is an open access article distributed under the terms and conditions of the Creative Commons Attribution (CC BY) license (http://creativecommons.org/licenses/by/4.0/). 